\title{
Vigilancia en red de los serotipos y la susceptibilidad antimicrobiana de Salmonella spp., Shigella spp. y Vibrio cholerae 01, 1997 - 1999
}

\author{
Nélida Muñoz ${ }^{1}$, Clara Inés Agudelo ${ }^{1}$, María Victoria Ovalle ${ }^{1}$, María Helena Realpe ${ }^{1}$ \\ y coordinadoras del programa en los Laboratorios de Salud Pública ${ }^{2}$ \\ ${ }^{1}$ Grupo de Microbiología, Instituto Nacional de Salud, Bogotá, D.C., Colombia \\ ${ }^{2}$ Edilma Jaramillo, LSP de Antioquia; Sandra Núñez, LSP de Bogotá; Martha Uzeta, Clínica del Niño, Bogotá; \\ Vianney Portilla, LSP de Santander; María del Pilar Crespo, Valle de Lili, Cali; María Elena Alvarez, LSP de \\ Caldas; Constanza Sabogal, LSP de Tolima; Mercedes Cano, LSP de Risaralda; María Claudia Rodríguez, \\ Hospital Erasmo Meoz, Cúcuta; María Eugenia Peláez, LSP de Córdoba; Lesli Bruzón, LSP de La Guajira; \\ Mileny Arregocés, LSP de Magdalena; Gloria Patricia Londoño, LSP de Amazonas; Gloria Inés Dussán, LSP \\ de Huila; Liliana Patiñc, LSP de Nariño; Alix Robinson, LSP de Arauca.
}

En 1997, el Grupo de Microbiología del INS estableció un programa en red con los Laboratorios de Salud Pública (LSP) del país y el apoyo de la OPS, para la vigilancia de los principales patógenos causantes de enfermedad diarreica aguda. El objetivo fue conocer los serotipos y los patrones de resistencia antimicrobiana de Salmonella spp., Shigella spp. y Vibrio cholerae O1. Los aislamientos fueron confirmados de acuerdo con los esquemas de identificación bioquímica y serológica estandarizados y la determinación de la susceptibilidad antimicrobiana se realizó por la técnica de difusión de disco (Kirby-Bauer). De 1997 a 1999, participaron 22 LSP con el envío de 976 aislamientos, $96 \%$ de origen clínico y $4 \%$ de alimentos; $34 \%$ Salmonella spp., $23 \%$ Shigella spp. y $42 \%$ V. cholerae O1. La distribución por serotipo de Salmonella fue $39 \%$ S. Enteritidis, $27 \%$ S. Typhimurium, $9 \%$ grupo E1, $5 \%$ S. Typhi y $20 \%$ otros serotipos; de los aislamientos de Shigella, $67 \%$ fueron S. grupo flexneri 2 a, $30 \%$ S. sonnei, $2 \%$ S. dysenteriae y $1 \%$ S. boydii. Para V. cholerae O1, 99\% fue serotipo Ogawa. La susceptibilidad antimicrobiana determinó que $56 \%$ de los aislamientos de Salmonella eran resistentes y $22 \%$ multirresistentes, con un patrón predominante de ampicilina, tetraciclina y trimetoprim-sulfa (SXT). De los aislamientos de Shigella, 97\% fueron resistentes y 57\% multirresistentes, con un patrón de ampicilina, tetraciclina, cloranfenicol y SXT. No se observaron cambios en la susceptibilidad de $V$. cholerae O1. Este estudio enfatiza la importancia de continuar con el programa de vigilancia, para conocer la epidemiología de la EDA en Colombia, darle un tratamiento óptimo a estas infecciones y poder diseñar programas para disminuir la diseminación de bacterias resistentes.

Palabras clave: Salmonella spp., Shigella spp., V. cholerae, EDA, serotipos, resistencia, vigilancia.

\section{Surveillance of serotypes and antimicrobial susceptibility of Salmonella spp., Shigella spp. and Vibrio cholerae 01, 1997-1999}

In 1997, a surveillance program to assess the main bacterial pathogens associated with acute diarrheal disease was initiated by the Microbiology Group of the Instituto Nacional de Salud in collaboration with the Public Health Laboratories (PHL) of the country and PAHO. The program objective was to identify the serotypes and antimicrobial resistance patterns of Salmonella spp., Shigella spp. and Vibrio cholerae 01 isolates. Twenty two PHL participated with a total of 976 isolates from 1997 to $1999 ; 96 \%$ were clinical isolates and $4 \%$ were food isolates. $34 \%$ were Salmonella spp., $23 \%$ Shigella spp., and $42 \%$ V. cholerae O1. From Salmonella isolates, $39 \%$ were serotype Enteritidis, 27\% Typhimurium, 9\% group E1, 5\% Typhi, and 20\% other serotypes. Among Shigella isolates, $67 \%$ were S. flexneri 2a, $30 \%$ S. sonnei, $2 \%$ S. dysenteriae and $1 \%$ S. boydii and $99 \%$ of the $V$. cholerae O1 isolates were serotype Ogawa. The study on the antimicrobial resistance determined that $56 \%$ of the Salmonella isolates were resistant and $22 \%$ of them were 
multiresistant, with the pattern ampicillin, tetracycline and trimethoprim sulfamethoxazole (SXT). $97 \%$ of Shigella isolates were resistant and $57 \%$ of them were multiresistant, with the pattern to tetracycline, ampicillin, SXT and chloramphenicol. The susceptibility of $V$. cholerae $\mathrm{O} 1$ isolates had not changed during the study period. This study ratifies the importance of surveillance on the epidemiology of these enteropathogens in Colombia. Based on this information it may be possible to treat these diseases and control the spread of antimicrobial bacterial resistance.

Key word: Salmonella spp., Shigella spp., V. cholerae, EDA, serotypes, resistance, surveillance.

El término 'vigilancia epidemiológica' aparece por primera vez a mediados de los años sesenta, cuando la Organización Mundial de la Salud constituyó una unidad con esa denominación. Los Centers for Disease Control and Prevention de Estados Unidos, propusieron, en 1986, el término 'vigilancia de la salud pública' con la siguiente definición: "la vigilancia en salud pública es la recolección sistemática y continua, análisis e interpretación de los datos de la salud, esenciales para la planificación, ejecución y evaluación de la práctica de la salud pública". El eslabón final de la cadena de la vigilancia es la aplicación de estos datos a la prevención y al control de las enfermedades (1).

De acuerdo con el sistema de vigilancia del Ministerio de Salud (SIS 12), Colombia ha presentado en los últimos 5 años, una tasa para la enfermedad diarreica aguda (EDA), mayor de 1.500 casos por 100.000 habitantes (2). El mismo sistema informa que $50 \%$ de los egresos hospitalarios por EDA, se deben a Salmonella spp. o Shigella spp.; sin embargo, no se conoce, de estos dos patógenos, los serotipos implicados en la enfermedad, ni los patrones de resistencia antimicrobiana (2). Es importante señalar que en nuestro medio los estudios relacionados con Salmonella implicada en EDA no señalan la distribución según el serotipo, sólo se conocen datos que la diferencian como Salmonella no Typhi y S. Typhi $(3,4)$.

La aplicación de marcadores fenotípicos tales como el serotipo y los patrones de susceptibilidad son la base para realizar estudios epidemiológicos

Correspondencia:

Nélida Muñoz

C. de M., INS

AA 80080

melidam@colnet.col.net.co

Recibido: 03/05/00; aceptado: 30/06/00 con los aislamientos bacterianos $(5,6)$, debido a que existen más de 2.435 serotipos en las subespecies de Salmonella, 49 en Shigella y 139 en Vibrio cholerae. Adicionalmente, la vigilancia por el laboratorio permite establecer la distribución de esos marcadores en los niveles local, regional, nacional e internacional, así como detectar la presencia de brotes de EDA y determinar el agente causal $(5,7)$.

A partir de 1997, el Grupo de Microbiología del Laboratorio Nacional de Referencia del Instituto Nacional de Salud (INS), inició con los Laboratorios de Salud Pública (LSP) del país un programa en red, para vigilar los principales agentes etiológicos de la EDA. El objetivo del programa es determinar los serotipos y la susceptibilidad antimicrobiana de Salmonella spp., Shigella spp. y $V$. cholerae O1. El programa ha contado con el apoyo de la Organización Panamericana de la Salud (OPS) y del Centro de Laboratorios para el Control de Enfermedades del Canadá (LCDC).

El objetivo del presente informe es dar a conocer el análisis epidemiológico de los resultados obtenidos de la vigilancia en red de los patógenos entéricos, en el período comprendido de enero de 1997 a diciembre de 1999.

\section{Materiales y métodos}

\section{Laboratorios de Salud Pública (LSP)}

Son los laboratorios de referencia en los departamentos que se encargaron de confirmar bioquímicamente todos los aislamientos obtenidos por los laboratorios clínicos y enviarlos al Grupo de Microbiología.

\section{Laboratorio de referencia}

El Grupo de Microbiología del INS, coordinador del programa en el nivel nacional, se encargó de 
capacitar a los LSP en el diagnóstico y la identificación por el laboratorio de los patógenos en estudio. Adicionalmente, se realizó la confirmación, la serotipificación y la determinación de la susceptibilidad antimicrobiana de los aislamientos enviados por los LSP.

\section{Datos de los pacientes y de los aislamientos}

La información de los pacientes se registró en un formato diseñado para tal fin, el cual contenía las variables demográficas y la procedencia; se determinó también si formaba parte de un brote. Sobre los aislamientos, se estableció la fuente, la identificación y los resultados del antibiograma, cuando se había realizado.

\section{Estudios bacteriológicos}

Todos los aislamientos se confirmaron bioquímicamente de acuerdo con los esquemas establecidos en el Laboratorio de Microbiología del INS $(8,9)$. La identificación serológica de las especies de Salmonella se realizó con el esquema de Kauffman-White (10), con el empleo de los antisueros: polivalente $O$ (grupos A-E) (INS) y los antisueros monovalentes de grupo $\mathrm{A}, \mathrm{B}, \mathrm{C} 1, \mathrm{C} 2$, C3, D1, D2, E1-E4, G1, G2 y H (Difco, Laboratorio de Salud Pública, Santiago de Chile y Fundación Fiocruz, Rio de Janeiro, Brasil) y flagelares H. Para Shigella, se utilizaron los antisueros de grupo (Difco) y de serotipo (Difco, Fundación Fiocruz, Rio de Janeiro, Brasil). La identificación serológica de $V$. cholerae 01 se realizó con antisueros polivalente y monovalentes, Inaba y Ogawa (Difco, Instituto Nacional de Salud).

\section{Susceptibilidad antimicrobiana}

Se determinó con la técnica de difusión de disco (Kirby-Bauer), de acuerdo con las recomendaciones del Comité Nacional para el Control de Estándares en el Laboratorio Clínico (NCCLS), tanto para su realización como para su interpretación (11).

Con los aislamientos de Salmonella y Shigella, se emplearon los siguientes sensidiscos (Difco u Oxoid) ampicilina $(10 \mu \mathrm{g})$, cloranfenicol $(30 \mu \mathrm{g})$, trimetoprim-sulfa $(1,25 / 23,75 \mu \mathrm{g})$, gentamicina (10 $\mu \mathrm{g})$, cefotaxima $(30 \mu \mathrm{g})$ y ciprofloxacina $(5 \mu \mathrm{g})$.

Con los aislamientos de $V$. cholerae 01 , se emplearon: ampicilina $(10 \mu \mathrm{g})$, cloranfenicol (30 $\mu \mathrm{g})$, trimetoprim-sulfa $(1,25 / 23,75 \mu \mathrm{g})$, eritromicina $(15 \mu \mathrm{g})$, tetraciclina $(30 \mu \mathrm{g})$ y ciprofloxacina $(5 \mu \mathrm{g})$.

\section{Análisis de los resultados}

Se hizo un análisis univariado, utilizando Epilnfo 6.0 (12). Se analizaron las variables de género, edad (estratificada en $<1$ año, 1 a 5,6 a 10,11 a 15 y $>15$ años), con respecto a la fuente y al serotipo encontrado.

\section{Resultados}

La participación de todos los LSP en la vigilancia de $V$. cholerae se inició en 1991 y aún se mantiene. En relación con la vigilancia de Salmonella spp. y Shigella spp., en 1997 participaron $8(22,8 \%)$ de los 35 LSP del país; en 1998, $13(37,1 \%)$, y en 1999, $15(42,9 \%)$.

De 1997 a 1999, se recibieron 976 aislamientos: $940(96,3 \%)$ de origen clínico y $36(3,7 \%)$ de alimentos. De los 940 aislamientos clínicos, 326 $(34,7 \%)$ fueron confirmados como Salmonella spp., 215 (22,9\%) Shigella spp. y $399(42,4 \%)$ V. cholerae O1. Los 36 aislamientos de alimentos fueron Salmonella spp.

En el cuadro 1 se consignó la procedencia de los aislamientos por regiones. Los Laboratorios de Salud Pública de Bogotá, Antioquia, Santander, Córdoba y Magdalena remitieron el mayor porcentaje de aislamientos, 27, 25, 8, 7 y $7 \%$, respectivamente.

De los 940 aislamientos clínicos, 194 (59,5\%) se recuperaron de pacientes del género masculino en el caso de Salmonella spp., $114(53,5 \%)$ en Shigella spp. y $246(62,6 \%)$ en V. cholerae 01. En la distribución por género de los pacientes, no hubo diferencia significativa en los tres enteropatógenos $(p>0,05)$. En la distribución por grupos de edad, se encontró que 127 (45,4\%) aislamientos de Salmonella spp. y $122(62,5 \%)$ de Shigella spp., eran de pacientes menores de 5 años con una media de 15,1 y 11,2 años, respectivamente, a diferencia de $V$. cholerae $\mathrm{O} 1$ donde $265(80,8 \%)$ se recuperaron de pacientes mayores de 15 años con una media de 26,3 (cuadro 2).

De los 326 aislamientos clínicos de Salmonella spp., $224(68,7 \%)$ se recuperaron de materia fecal, 
Cuadro 1. Procedencia de los aislamientos de Salmonella spp., Shigella spp. y Vibrio cholerae O1, confirmados de enero de 1997 a diciembre de 1999.

\begin{tabular}{|c|c|c|c|c|c|c|c|c|c|}
\hline \multirow[t]{2}{*}{ Región } & \multirow[t]{2}{*}{ Departamento } & \multicolumn{2}{|c|}{ Salmonella spp. } & \multicolumn{2}{|c|}{ Shigella spp. } & \multicolumn{2}{|c|}{ V. cholerae 01} & \multicolumn{2}{|c|}{ Total } \\
\hline & & $\mathrm{n}$ & $(\%)$ & $n$ & $(\%)$ & $\mathrm{n}$ & $(\%)$ & n & $(\%)$ \\
\hline \multirow[t]{5}{*}{ Occidente } & Antioquia & 108 & $(29,8)$ & 26 & $(12,1)$ & 106 & $(26,6)$ & 240 & $(24,6)$ \\
\hline & Caldas & 11 & $(3,0)$ & & & 3 & $(0,8)$ & 14 & $(1,4)$ \\
\hline & Chocó & & & & & 1 & $3(0,8)$ & 9 & $(0,9)$ \\
\hline & Risaralda & 6 & $(1,7)$ & 12 & $(5,6)$ & & & 18 & $(1,8)$ \\
\hline & Valle & 13 & $(3,6)$ & 8 & $(3,7)$ & 2 & $(0,5)$ & 23 & $(2,4)$ \\
\hline \multirow[t]{6}{*}{ Centro-oriente } & Bogotá & 136 & $(37,6)$ & 127 & $(59,1)$ & & & 263 & $(26,9)$ \\
\hline & Cundinamarca & & & 2 & $(0,9)$ & 6 & $(1,5)$ & 8 & $(0,8)$ \\
\hline & Huila & 2 & $(1,0)$ & & & & & 2 & $(0,2)$ \\
\hline & Norte de Santander & 11 & $(3,0)$ & & & 26 & $(6,5)$ & 37 & $(3,8)$ \\
\hline & Santander & 41 & $(11,3)$ & 35 & $(16,3)$ & 2 & $(0,5)$ & 78 & $(8,0)$ \\
\hline & Tolima & 13 & $(3,6)$ & 2 & $(0,9)$ & 4 & $(1,0)$ & 19 & $(1,9)$ \\
\hline \multirow[t]{7}{*}{ Costa Atlántica } & Atlántico & 1 & $(0,3)$ & & & 9 & (2.3) & 10 & $(1.0)$ \\
\hline & Bolívar & 1 & $(0,3)$ & & & 29 & $(7,3)$ & 30 & $(3,1)$ \\
\hline & Cesar & & & & & 25 & $(6,3)$ & 25 & $(2,6)$ \\
\hline & Córdoba & & & & & 66 & $(16,5)$ & 66 & $(6,8)$ \\
\hline & La Guajira & 2 & $(0,6)$ & & & 43 & $(10,8)$ & 45 & $(4,6)$ \\
\hline & Magdalena & 2 & $(0,6)$ & 2 & $(0,9)$ & 60 & $(15,0)$ & 64 & $(6,6)$ \\
\hline & Sucre & & & & & 9 & $(2,3)$ & 9 & $(0,9)$ \\
\hline Amazonia & Amazonas & 3 & $(0,8)$ & 1 & $(0,5)$ & 4 & $(1,0)$ & 8 & $(0,8)$ \\
\hline \multirow[t]{2}{*}{ Orinoquia } & Arauca & 5 & $(1,4)$ & & & & & 5 & $(0,5)$ \\
\hline & Meta & 1 & $(0,3)$ & & & 1 & $(0,3)$ & 2 & $(0,2)$ \\
\hline \multicolumn{2}{|l|}{ Total } & \multicolumn{2}{|c|}{$362(100,0)$} & \multicolumn{2}{|c|}{$215(100,0)$} & \multicolumn{2}{|c|}{$399(100,0)$} & 976 & $(100,0)$ \\
\hline
\end{tabular}

Aislamientos clínicos, $n=940$

Aislamientos de alimentos, $n=36$

$80(24,6 \%)$ de procesos invasores (61 de sangre y 19 de LCR y otros líquidos corporales estériles) y $22(6,7 \%)$ de otras fuentes (orina, flujo vaginal, secreción de herida). Todos los aislamientos de Shigella spp. y V. cholerae $\mathrm{O} 1$ fueron de materia fecal.

La distribución por serotipos de los 326 aislamientos clínicos de Salmonella spp. indicó que $134(41,1 \%)$ eran Salmonella Enteritidis, 90 (27,6\%) Salmonella Typhimurium, $23(7,1 \%)$ Salmonella Grupo E1, 18 (5,5\%) Salmonella Typhi: $61(18,7 \%)$ aislamientos correspondían a 33 serotipos diferentes de 1 a 5 aislamientos cada uno.

Al estratificar por serotipos los 80 aislamientos invasores de Salmonella, se encontró que 37 $(46,2 \%)$ fueron serotipo Enteritidis, $16(20,0 \%)$ Typhi, 11 (13,8\%) Typhimurium; 16 (20,0\%) aislamientos correspondían a 14 serotipos diferentes.
La media de la edad de los 90 pacientes con $S$. Typhimurium fue de 11,6 años; de ellos $52(57,8 \%)$ eran menores de 5 años y la media de los 134 con $S$. Enteritidis fue de $17,7 \mathrm{y}$, de ellos, 37 $(27,6 \%)$ se asociaron con el grupo de menores de 5 años $(p=0,006)$.

De los 36 aislamientos de alimentos, $9(25,0 \%)$ fueron serotipo Typhimurium, 9 (25,0\%) grupo E1, $6(16,7 \%)$ Enteritidis, $3(8,3 \%)$ grupo B y $9(25,0 \%)$ aislamientos correspondían a 9 serotipos diferentes.

Adicionalmente, el sistema de vigilancia permitió detectar la presencia de tres brotes de intoxicación alimenticia, uno en Santander (14 aislamientos) causado por Salmonella Uganda y uno en Cundinamarca ( 6 aislamientos) por Salmonella Enteritidis. Un tercer brote se detectó en Arauca (3 aislamientos) causado por Salmonella Newport; de ninguno de ellos se conoció la fuente. 
Cuadro 2. Distribución por grupos de edad de pacientes con aislamientos clínicos de Salmonella spp., Shigella spp. y Vibrio cholerae O1, confirmados de enero de 1997 a diciembre de $1999\left(n=803^{\star}\right)$.

\begin{tabular}{lrrrrrrr}
\hline \multirow{2}{*}{$\begin{array}{c}\text { Grupo } \\
\text { de edad } \\
\text { (años) }\end{array}$} & \multicolumn{6}{c}{ Salmonella spp. Shigella spp. . cholerae 01 } \\
\cline { 2 - 8 } & $\mathbf{n}$ & $(\%)$ & $\mathbf{n}$ & $(\%)$ & $\mathbf{n}$ & $(\%)$ \\
\hline$\leq 1$ & 85 & $(30,4)$ & 43 & $(22,1)$ & 7 & $(2,1)$ \\
$2-5$ & 42 & $(15,0)$ & 79 & $(40,4)$ & 37 & $(11,3)$ \\
$6-10$ & 26 & $(9,2)$ & 25 & $(12,8)$ & 19 & $(5,8)$ \\
$11-15$ & 20 & $(7,2)$ & 4 & $(4,0)$ & & \\
$>15$ & 107 & $(38,2)$ & 44 & $(22,6)$ & 265 & $(80,8)$ \\
Total & 280 & $(100)$ & 195 & $(100)$ & $\mathbf{3 2 8}$ & $(100)$ \\
\hline
\end{tabular}

*137 aislamientos no tenían dato de edad

De los 215 aislamientos del género Shigella, 143 $(66,5 \%)$ fueron $S$. grupo flexneri ( $87 \%$ serotipo $2 a)$, $64(29,8 \%)$ S. grupo sonnei, $4(1,9 \%)$ S. grupo dysenteriae (serotipos de 1 al 7 ), $3(1,4 \%)$ S. grupo boydii (serotipos del 1 al 7 ) y $1(0,5 \%)$ no serotipificable. Al relacionar los serogrupos de Shigella spp. con la edad de los pacientes se encontró que la media de la edad para $S$. flexneri fue de 11,0 y para $S$. sonnei 9,8 , sin encontrarse diferencia significativa entre los dos serogrupos.

De los 399 aislamientos de V. cholerae 01, 395 (99\%) fueron serotipo Ogawa y 4 (1\%) serotipo Inaba.

Del total de aislamientos de Salmonella spp., 118 $(32,5 \%)$ presentaron resistencia a tetraciclina, 94 $(26 \%)$ a ampicilina, $86(23,8 \%)$ a SXT, $14(3,9 \%)$ a cloranfenicol, $9(2, \%)$ a cefotaxima y $5(1,4 \%)$ a gentamicina; todos los aislamientos fueron susceptibles a ciprofloxacina. En resumen, 201 $(55,5 \%)$ presentaron resistencia a uno o varios de los antimicrobianos estudiados y $80(22,4 \%)$ fueron multirresistentes (resistencia a 3 o más familias de antimicrobianos). El análisis del patrón de multirresistencia por serotipos estableció que $67 / 99(67,7 \%)$ aislamientos de $S$. Typhimurium fueron multirresistentes, comparado con 14/263 $(5,3 \%)$ de los otros serotipos $(p<0,001)$. El patrón de multirresistencia que con mayor frecuencia se observó fue ampicilina, tetraciclina y SXT (cuadro 3).

El análisis de multirresistencia de los aislamientos de Salmonella spp. por procedencia se realizó con los departamentos con más de 10 de aislamientos
Cuadro 3. Distribución de los 80 aislamientos de Salmonella spp. por patrón de multirresistencia, serotipo y procedencia 1997 - 1999 ( $n=362)$.

\begin{tabular}{|c|c|c|c|c|}
\hline \multirow[t]{2}{*}{ Patrón } & \multicolumn{2}{|c|}{ Serotipo } & \multicolumn{2}{|c|}{ Total } \\
\hline & $\begin{array}{c}\text { Typhimurium } \\
\mathrm{n}\end{array}$ & Otros* & $\mathbf{n}$ & $\%$ \\
\hline \multicolumn{5}{|l|}{ Amp, Te, $\mathrm{SXT}$} \\
\hline Antioquia & 35 & 3 & & \\
\hline Bogotá & 17 & 1 & & \\
\hline Santander & 2 & & & \\
\hline Tolima & 1 & & & \\
\hline Valle & 1 & & & \\
\hline Otros & 2 & 2 & & \\
\hline Subtotal & 58 & 6 & 64 & 17,7 \\
\hline \multicolumn{5}{|c|}{ Otros patrones ${ }^{\star \star}$} \\
\hline Antioquia & 6 & 1 & & \\
\hline Bogotá & & 2 & & \\
\hline Santander & 3 & 2 & & \\
\hline Otros & 2 & & & \\
\hline Subtotal & 11 & 5 & 16 & 4,4 \\
\hline Total & 69 & 11 & 80 & 22,1 \\
\hline
\end{tabular}

* Serotipos Infantis, Grupo B, Enteritidis, Saint Paul,

** Amp, Te, SXT, CTX

Amp, Te, SXT, CTX, GM

Amp, Te, SXT, C

Amp, Te, C

Amp, SXT, C

$\mathrm{Amp}=$ ampicilina, $\mathrm{Te}=$ tetraciclina, $\mathrm{SXT}=$ trimetoprim sulfametoxasol, $\mathrm{CTX}=$ cefotaxima, $\mathrm{GM}=$ gentamicina $\mathrm{C}=$ cloranfenicol

y señaló que 45/108 (51\%) aislamientos de Antioquia eran multirresistentes, comparado con $21 / 136(15,4 \%)$ de Bogotá, $2 / 13(15,4 \%)$ del Valle, $1 / 13(7,7 \%)$ del Tolima y $6 / 41(1,5 \%)$ de Santander $(p<0,001)$. Los departamentos de Caldas y Norte de Santander no tuvieron aislamientos con multirresistencia.

Del total de aislamientos de Shigella spp., 202 $(94 \%)$ presentaron resistencia a la tetraciclina, 172 $(79,9 \%)$ a SXT, $144(66,9 \%)$ a ampicilina, 117 $(54,2 \%)$ a cloranfenicol, $5(2,4 \%)$ a cefotaxima y $1(0,5 \%)$ a gentamicina; todos los aislamientos fueron susceptibles a ciprofloxacina. En resumen, $204(94,9 \%)$ presentaron resistencia a uno o varios de los antimicrobianos estudiados y $122(56,7 \%)$ fueron multirresistentes. El cuadro 4 muestra la distribución de los aislamientos de Shigella spp., por patrón de multirresistencia, serogrupo y procedencia; $208(96,7 \%)$ presentaron algún tipo de resistencia y $122 / 215(56,7 \%)$ presentaron uno 
Cuadro 4. Distribución de los 122 aislamientos de Shigella spp. por patrón de multirresistencia, serogrupo y procedencia 1997 - 1999.

\begin{tabular}{|c|c|c|c|c|}
\hline \multirow[t]{2}{*}{ Patrón } & \multicolumn{2}{|l|}{ Serogrupos } & \multirow{2}{*}{$\begin{array}{l}\text { Total } \\
\mathbf{n}\end{array}$} & \\
\hline & $\begin{array}{c}\text { flexneri } 2 a \\
n\end{array}$ & otros & & $\%$ \\
\hline
\end{tabular}

\begin{tabular}{|c|c|c|c|c|c|}
\hline Amp, Te, C, SXT & & & & & \\
\hline Bogotá & 47 & & & & \\
\hline Santander & 22 & & & & \\
\hline Antioquia & 6 & 1 & 1 & & \\
\hline Otros & 9 & & 1 & & \\
\hline Risaralda & & 1 & & & \\
\hline Subtotal & 84 & 2 & 2 & 88 & 40,9 \\
\hline Amp, Te, C & & & & & \\
\hline Bogotá & 14 & & & & \\
\hline Antioquia & 1 & & & & \\
\hline Subtotal & 18 & 1 & & 19 & 8,8 \\
\hline Otros patrones* & & & & & \\
\hline Bogotá & 4 & 3 & & & \\
\hline Antioquia & 3 & & & & \\
\hline Santander & 2 & & & & \\
\hline Otros & 1 & 2 & & & \\
\hline Subtotal & 10 & 5 & & 13 & 6,0 \\
\hline Total & 112 & 8 & 2 & 122 & 56,7 \\
\hline
\end{tabular}

Amp $=$ ampicilina, $\mathrm{Te}=$ tetraciclina, $\mathrm{SXT}=$ trimetoprim sulfametoxasol, $\mathrm{C}=$ cloranfenicol

*Amp, Te, SXT

Amp, Te, SXT,C, Ctx

de los cuatro patrones de multirresistencia, de los cuales el más frecuente fue ampicilina, tetraciclina, cloranfenicol y SXT (40,9\%); S. flexneripresentó el mayor porcentaje de multirresistencia 112/143 $(78,3 \%)$, comparado con $10 / 72(13,8 \%)$ de otros serogrupos $(p<0,001)$.

El análisis de multirresistencia por procedencia se realizó con los departamentos que tenían más de 10 aislamientos. De 35 aislamientos de Shigella spp. procedentes de Santander, $27(77,1 \%)$ fueron multirresistentes comparados con $68 / 127(53,5 \%)$ de Bogotá, 11/26 (42,3\%) de Antioquia y 1/12 $(8,3 \%)$ de Risaralda $(p=0,05$ y $p=0,007)$.

Dado que el comportamiento de los aislamientos de $V$. cholerae $\mathrm{O} 1$ frente a los antibióticos estudiados, presentó anualmente una marcada variabilidad, los resultados de la susceptibilidad antimicrobiana de cada año se analizaron por separado. La distribución de los aislamientos resistentes por departamento y antibiótico, correspondiente a 1997 y ya fue publicada (13). En 1998, se encontró que 3/11 (2,7\%) aislamientos presentaron resistencia a ampicilina y $2(1,8 \%)$ a SXT, tetraciclina y cloranfenicol; todos procedían del departamento de Córdoba. Los 6 aislamientos de 1999 fueron sensibles a los antimicrobianos estudiados.

\section{Discusión}

Este sistema de vigilancia en red con los LSP, ha producido resultados que han permitido conocer los serotipos más frecuentes y los patrones de resistencia que presentan estos enteropatógenos, así como también realizar el análisis clínicoepidemiológico. Adicionalmente, mantener la vigilancia del cólera por el laboratorio, la cual se inició en 1991 con la aparición de la epidemia.

Los LSP (20/35) que participaron con el mayor número de aislamientos representan $66 \%$ de la población ya que éstos corresponden a los departamentos del país más densamente poblados.

En el análisis de la distribución de los aislamientos, con respecto al género de los pacientes infectados con Salmonella spp. $y$ Shigella spp., no se observaron diferencias significativas, lo que hace pensar que el género de los pacientes no condiciona su riesgo para la infección con estos patógenos. En la infección con $V$. cholerae 01 , el número de mujeres fue inferior al de hombres, comportamiento que se mantiene igual al observado durante la epidemia. (14).

Con relación a la edad, en esta vigilancia, el grupo afectado con mayor frecuencia con Salmonella spp. fue el de menores de 15 años y, entre ellos, el grupo de menores de 1 año $(45,4 \%)$. Esto indica que los niños son más susceptibles que los adultos a las infecciones por Salmonella spp. y esto podría estar relacionado con la inmadurez de su sistema inmune $(4,15,16)$. La gastroenteritis por Shigella spp. se encontró principalmente en niños menores de 5 años; varias causas pueden explicar este predominio infantil como lo son la fácil transmisión orofecal a través de las manos dada la falta de hábitos higiénicos en los niños de edad preescolar, así como las deficiencias en los 
mecanismos de inmunidad intestinal en esta población $(17,18)$. En contraste, en el cólera, el grupo de los mayores de 10 años continúa siendo el más afectado, comportamiento dado por una tasa baja de infección de tipo endémico (19).

La EDA causada por Salmonella spp. no tífica fue la más frecuente en nuestro país, encontrándose que los serotipos asociados con el mayor número de casos de infección humana fueron $S$. Enteritidis y $S$. Typhimurium. Esta distribución es similar a la encontrada en México y Brasil pero diferente a la de Argentina y Chile en donde el serotipo es $S$. Infantis (Informe de la evaluación de programa de resistencia antimicrobiana de bacterias enteropatógenas, proyecto colaborativo $\mathrm{PAHO}$ LCDC, Chile 2000). En Shigella, el grupo prevalente fue $S$. flexneri serotipo $2 a$, comportamiento similar al encontrado en otros países de Latinoamérica como Argentina, Brasil, Chile, Perú y México. En los países desarrollados, S. sonnei, es el grupo predominante (18). El serotipo de $V$. cholerae 01 circulante en nuestro país y en Latinoamérica sigue siendo Ogawa.

El incremento de la resistencia a los antibióticos de los serotipos de Salmonella spp. es un problema importante en el ámbito mundial. Durante la pasada década, varios serotipos han demostrado una alarmante habilidad para adquirir altos niveles de resistencia frente a los antibióticos de relevancia clínica: ampicilina, SXT y cloranfenicol. La resistencia en Salmonella spp. a los antibióticos en general está codificada por plásmidos, pero también puede ser el resultado de una mutación espontánea de un gen cromosómico como respuesta a la presión selectiva que ejerce el uso de antibióticos en humanos o en medicina veterinaria (20).

El resultado de la vigilancia señaló una frecuencia de resistencia de $25 \%$ a ampicilina, SXT y tetraciclina; esta resistencia es comparable a la encontrada en España (24\%) (15), pero menor a la informada por Mattar (21) en aislamientos de niños colombianos, donde la resistencia a la ampicilina fue de $67,5 \%$, la que pudo estar influida por el tamaño de la muestra analizada (40 aislamientos) y, por tanto, no ser representativa.

Con relación a los otros antimicrobianos, Salmonella spp. presentó una alta sensibilidad a cloranfenicol ( $96 \%$ ) y a ciprofloxacina $(100 \%)$. Sin embargo, es importante señalar que el cloranfenicol no tiene una acción bactericida para Salmonella spp. y, por tanto, puede estar asociada con una alta tasa de recaidas, mayor que la ocasionada por la ampicilina, pero esta última no elimina el estado de portador gastrointestinal. El uso de las quinolonas resulta una importante estrategia en salud pública, dado que tienen la habilidad de penetrar en la vesícula biliar y en el hígado y son más efectivas para erradicar Salmonella spp. en los portadores crónicos (22).

El serotipo $S$. Typhimurium presentó un patrón de multirresistencia característico, el cual no se observó en ningún otro serotipo (17).

La resistencia antimicrobiana de los aislamientos de Shigella spp. fue mayor al compararla con los aislamientos de Salmonella spp.. El 97\% de los aislamientos de Shigella spp. presentaron algún tipo de resistencia a ampicilina, SXT, tetraciclina y cloranfenicol y el patrón de multirresistencia frente a los antibióticos mencionados se observó principalmente en S. flexnerii serotipo 2a. Este patrón es similar al encontrado en Bangladesh (16) y es indicativo del mal uso de los antibióticos en muchos países en vìas de desarrollo. Todos los aislamientos fueron altamente sensibles a las fluoroquinolonas, gentamicina y cefotaxima.

Para el manejo de las infecciones invasoras por Salmonella spp. y Shigella spp., se debe tener en cuenta la presencia de los serotipos de $S$. flexnerii y $S$. Typhimurium multirresistentes; por tanto, es de particular interés confirmar la susceptibilidad frente al tratamiento establecido, mediante pruebas de susceptibilidad específicas.

La prevalencia de aislamientos resistentes de $\mathrm{V}$. cholerae 01 fue diferente en cada año del estudio; esto se debe a que la resistencia es mediada en gran parte por plásmidos y debido a que estos microorganismos no los portan en forma permanente, los patrones de resistencia varían (17). En el último año, el comportamiento fue estable frente a los antibióticos de elección como lo son la eritromicina y la tetraciclina, a excepción de 2 casos provenientes del departamento de Córdoba.

Este estudio enfatiza la importancia de continuar con el programa de vigilancia de la resistencia de 
estos enteropatógenos en Colombia, con el fin de conocer la epidemiología de la EDA a escala nacional, para darle un tratamiento óptimo a las infecciones, proponer estrategias para disminuir la diseminación de bacterias resistentes a los antibióticos, controlar las enfermedades endémicas y detectar la aparición de brotes. Por tanto, es de vital importancia que todos los LSP del país sirvan de apoyo al Sistema General de Seguridad Social en Salud, para la vigilancia de estas y otras patologías de importancia en salud pública.

\section{Referencias}

1. Tello O, Heras CA, del Amo IP, Martínez JF. Vigilancia de la salud pública. En: Martínez F, Antó JM, Castellanos PL, Gili M, Morset P, Navarro V, editores. Salud pública. Madrid, España: McGraw-Hill Interamericana; 1997.

2. Cáceres DC, Acosta J. Evaluación de la situación de cólera en Colombia. Inf Quinc Epidemiol Nac 1998;4:18.

3. Jaramillo E, Estrada S, Ospina S. Etiología de la enfermedad diarreica aguda (EDA) de origen bacteriano utilizando un protocolo estandarizado de laboratorio. Infectio 1999;3:95-9.

4. Bernal C, Chichilla CF, Jaimes LE. Salmonella spp. en niños hospitalizados por enfermedad diarreica. Un estudio de casos y controles. latreia 1998;11:56-9.

5. Usera MA, Cano RX, Echeita A. Análisis de los serotipos de Salmonella sp. aislados en España en el período 19881992. Enferm Infecc Microbiol Clin 1995; 13:138-45.

6. Helderbg CW, Angulo FJ, White KE, Langkop CW, Schell WI, Stobierski MG, et al. Outbreaks of Salmonellosis associated with eating uncooked tomatoes: implications for public health. Epidemiol Infect 1999;122:385-93.

7. Taunay AE, Fernandes SA, Tavechio AT, Neves BC, Dias AM, Irino $\mathrm{K}$. The role of the public health laboratory in the problem of Salmonellosis in Sao Paulo, Brazil. Rev Inst Med Trop Sao Paulo 1996;38:119-27.

8. Gray LD. Escherichia, Salmonella, Shigella and Yersinia. In: Murray PR, Baron EJ, Pfaller MA, Tenover FC, Yolken $\mathrm{RH}$, editores. Manual of clinical microbiology. Sixth Edition. Washington, D.C.: American Society for Microbiology; 1995. p.450-65.

9. McLaughlin JC. Vibrio. In: Murray PR, Baron EJ, Pfaller MA, Tenover FC, Yolken RH, editores. Manual of clinical microbiology. Sixth Edition. Washington, D.C.: American Society for Microbiology; 1995. p.465-76.
10. World Health Organization Collaborating Centre for Reference and Research on Salmonella. Antigenic formulae of the Salmonella serovars. Paris: WHO International Salmonella Center, Institut Pasteur; 1997.

11. National Committee for Clinical Laboratory Standards. Performance standars for antimicrobial testing; eight informational supplement. NCCLS document M100-S8; 18. Wayne, Pennsylvania: NCCLS; 1998.

12. Dean AG, Dean JA, Columbier D, Burton AH, Brendel KA, Smith DC, et al. Epilnfo versión 6.0: a word processing, data-base and statistics program for epidemiology on microcomputers. Atlanta, Georgia: Centers for Disease Control and Prevention; 1994.

13. Agudelo $\mathrm{Cl}$, Castañeda E, Gómez L, Parrado CY. Informe de los primeros aislamientos clínicos de Vibrio cholerae 01 resistentes a los antimicrobianos. Inf Quinc Epidemiol Nac 1997;2:81.

14. Vargas Cl, Gómez L. Informe mensual de los aislamientos de Vibrio cholerae $\mathrm{O} 1$ realizado por los servicios de salud. Enero-noviembre de 1995. IQCB (INS) 1995; 1:45-7.

15. Lee SC, Yang PH, Shieh WB, Lasserre R. Bacteremia due to non-Typhi Salmonella: analisis of 64 cases and review. Clin Infect Dis 1994;19:693-6.

16. Baquero F. Antibiotic resistence in Spain: What can be done? Clin Infect Dis 1996;23:819-25.

17. Sack RB, Rahman M, Yunus M, Khan EH. Antimicrobial resistance in organisms causing diarrheal disease. Clin Infect Dis 1997;24(Suppl.1):S102-5.

18. Morera MA, Espejo E, Coll P, Simón M, Uriz MS, Llovet $\mathrm{T}$, et al. Epidemic outbreak of shigellosis following water intake. Enfer Infect Microbiol Clin 1995;13:160-5.

19. Centers for Disease Control and Prevention. Métodos del laboratorio para el diagnóstico de Vibrio cholerae. Atlanta, Georgia: CDC/NCID OPS; 1994.

20. Richmond $\mathbf{M H}$, Linton KB. The use of tetracycline in the community and its possible relation to the excretion of tetracycline-resistant bacteria. J Antimicrob Chemother 1980;6:33-41.

21. Mattar S, Vásquez E. Antimicrobial susceptibility of enteropathogenic bacteria causing infectious diarrhoea in pediatric patients from Colombia. Medical Science Research 1998;26:393.

22. Bryan JP, Rocha $\mathbf{H}$, Scheld WM. Problems in salmonellosis: rationale for clinical trials with newer Blactam agents and quinolones. Rev Infect Dis 1986;8:18997. 